\title{
Exercise-induced ventricular dysfunction in hypertrophic cardiomyopathy: stunning by any other name?
}

\author{
Houman Ashrafian, Hugh Watkins
}

Hypertrophic cardiomyopathy (HCM) is a common cardiac genetic disorder with a prevalence of $1 / 500$ and is caused principally by mutations in genes encoding proteins of the cardiac sarcomere (eg, $\beta$ cardiac myosin heavy chain and cardiac troponin T). ${ }^{1}$ Although of substantial scientific importance, HCM is best recognised for being the most common cause of sudden cardiac death (SCD) in the young, especially in young athletes, accounting for up to one-third of all such deaths in the US. ${ }^{2}$ HCM might be expected to impair exercise capacity, for example through left ventricular hypertrophy $(\mathrm{LVH})$ and diastolic ventricular dysfunction; however, some HCM patients not only tolerate exercise well, but indeed excel athletically. This is surprising given that even HCM patients without LVH exhibit subtle systolic and diastolic abnormalities. Additionally, approximately one-third of HCM patients exhibit abnormal blood pressure (bp) responses during maximal treadmill exercise (ie, a flat blood pressure response, or a fall in blood pressure), which portends a poor prognosis. ${ }^{3}$ Ultimately, exercise in HCM is a matter of considerable concern, as many of the cases of SCD in HCM are associated with exertion. ${ }^{2}$ Exerciseinduced SCD thus represents the rationale, albeit controversially, for preparticipation cardiac screening and exclusion of affected athletes from sporting activity. ${ }^{5}$

\section{WHAT THEN IS THE IMPACT OF EXERCISE IN HCM?}

Sakata et al address this question in this issue of (see page 1282) by proposing that exercise-induced LV systolic dysfunction (LVSD) occurs more frequently in HCM patients with cardiac troponin gene mutations than in those without troponin mutations. ${ }^{6}$ While this observation is

Correspondence to: Drs Houman Ashrafian and Hugh Watkins, Department of Cardiovascular Medicine,

University of Oxford, John Radcliffe Hospital, Oxford OX3 9DU; houman.ashrafian@cardiov.ox.ac.uk potentially of great interest, it is not without methodological limitations. First, there are marked differences between the two patient groups other than, but potentially not independent of, their genetics. These include marked differences in: number of patients recruited (10 vs 42), age (43.6 vs 53.4 years old), family history of HCM $(100 \%$ vs $23.8 \%)$ and family history of SCD $(100 \%$ vs $16.7 \%)$ in troponin and non-troponin mutation respectively. Ascertainment bias is a well-recognised confounding feature of HCM genetic studies ${ }^{1}$; additionally, differences in the age and family history of patients recruited in each group are pertinent confounders to this study. However, the greatest limitation of this work is apparent in Sakata et al's fig 2, which suggests that, while overall differences exist between the two groups, a proportion of non-troponin mutation patients also exhibit exercise-induced LVSD. This can be interpreted to mean that a subgroup of non-troponin mutation patients also exhibit a physiology akin to that of troponin mutation patients. It is striking that so many in the non-troponin mutation group are older and do not have known familial HCM, suggesting a mixed group of "phenocopies" as well as some true sarcomeric HCM. Thus the data are compatible with a different interpretation - that exercise-induced LVSD is characteristic of typical familial sarcomeric HCM per se. Rather like an abnormal bp response, exercise-induced LVSD may more likely be a manifestation of aberrant HCM physiology than a manifestation of any given gene mutation. Subgroups with sarcomeric HCM in the non-troponin mutant group would be statistically subsumed by the greater mass of patients with no change in their LV function. While the consequences of confounders are ill-defined, they may nevertheless limit the generalisability of these findings.

Another limitation of this study is the use of supine ergometer exercise and radionuclide ventricular function monitoring with a cadmium telluride detector (VEST). Since abnormal bp responses during exercise represent a prognostic indicator in $\mathrm{HCM},{ }^{4}$ there has been extensive interest in the haemodynamic determinants of this phenomenon. Pertinent to the present study, some investigators suggest that abnormal bp responses are due to a profound impairment of cardiac output augmentation (ie, systolic function) on exercise. ${ }^{7}$ Other investigators have disputed this finding ${ }^{8}$ by suggesting that abnormal bp responses are due to an exaggerated fall in systemic vascular resistance due to vasodilation in nonexercising vascular beds instead of the "normal" vasoconstrictor response. ${ }^{910}$ Both these aberrations would incur abnormal bp responses, though through different mechanisms. These discrepancies may relate, in part, to limitations of VEST. While VEST has been used in numerous studies, there are potential methodological difficulties (eg, not being able to ensure that the detector faithfully follows the ventricle during exercise despite visual adjustment pre and post-exercise); it has not been validated against invasive studies. ${ }^{8}$ These limitations may have been further complicated by the use of VEST to determine supine exercise haemodynamics. These limitations notwithstanding, previous VEST studies in HCM attest to the common physiology of HCM patients, which is similar even in obstructive and non-obstructive disease..$^{7112}$ This common physiology of HCM reinforces the proposal that at least some of the non-troponin mutation group with seemingly different physiology may not have had sarcomeric HCM but may instead have been phenocopies.

\section{WHAT ARE THE CLINICAL CONSEOUENCES OF THIS STUDY?}

There is a significant clinical need for better surrogate markers for HCM prognosis, since identifying high-risk HCM patients for aggressive prophylactic therapy (eg, implantable cardioverter defibrillators) is at present imprecise. ${ }^{4}$ While this study suggests that a subgroup of HCM patients exhibit severe physiological aberrations, at least at present it does not support exercise-induced LVSD as a clinical test for prognostication. In a prospective exercise echocardiographic study of 35 HCM patients, exercise-induced LVSD at baseline was associated with clinical deterioration (eg, by deteriorating resting LV function) and worse clinical outcome at 6.4 years' follow-up (fig 1). Do the 

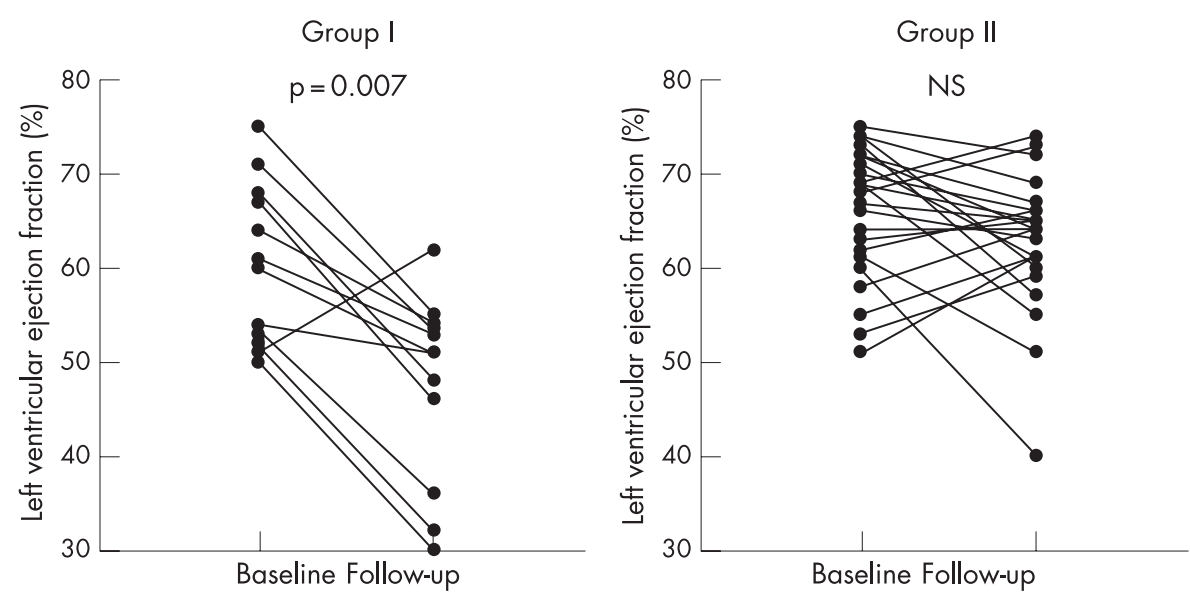

Figure 1 Left ventricular ejection fraction at baseline and at last follow-up evaluation in patients of group I (EILVSD) and group II (Non-EILVSD). This study demonstrates that patients with exerciseinduced left ventricular systolic dysfunction (EILVSD) show a markedly increased potential for deteriorating ventricular systolic function at 6.4 years' follow-up. While this study was not powered for formal clinical endpoints (eg, mortality or sudden cardiac death), intriguingly, though not conclusively, these events appeared to be increased in Group I. ${ }^{13}$ Reprinted with permission from International Journal of Cardiology, vol 1, Francesco Pelliccia, Cumulative exercise-induced left ventricular systolic and diastolic dysfunction in hypertrophic cardiomyopathy, pp 1-2, copyright Elsevier (2008).

patients identified by Pelliccia et $a l^{13}$ in their Group 1, with exercise-induced LVSD and a poorer prognosis, overlap with the troponin mutation patients identified with exercise-induced LVSD by Sakata et al? While both studies are of great interest, bearing in mind the limitations of study size, power and methodology, Sakata et al, to their credit, are circumspect about the mechanistic and clinical applicability of their study pending much larger studies.

\section{WHAT ARE THE MECHANISTIC CONSEQUENCES OF THIS STUDY?}

Whether or not the specificity of these findings for troponin mutations is confirmed or extended to a broader group of other sarcomeric HCM patients with aberrant physiology, the study by Sakata et al raises a number of as yet unexplored novel mechanistic questions. There is extensive precedent for episodes of exercise-induced contractile dysfunction in ischaemic heart disease; this has broadly been termed myocardial stunning. ${ }^{14}$ This persisting contractile dysfunction resulting from ischaemia has been attributed to energy deficiency as manifested by the decreasing myocardial adenine nucleotide pool (ie, $[\mathrm{ATP}+\mathrm{ADP}+\mathrm{AMP}])$ and the ensuing alterations in oxygen radicals and calcium homeostasis. ${ }^{15} \mathrm{We}$ and others have proposed, with substantial supporting evidence, that HCM is a disease of energy deficiency. ${ }^{1}$ In HCM, could sarcomeric rather than ischaemic energy deficiency be exacerbated during exercise sufficiently to cause systolic dysfunction? If so, are mechanisms similar to that enacted during ischaemic stunning pertinent to HCM-induced exercise-induced LVSD (HCM-induced "stunning")? It is plausible that some HCM patients, as identified by Sakata et al, experience particularly extreme energy deficiency as their mutations appear to be especially energetically profligate. ${ }^{16}{ }^{17}$ Furthermore, there are also other similarities between ischaemia and HCM. Severe microvascular dysfunction is found in HCM, manifesting as impaired coronary flow reserve in hypertrophied segments, and this may mediate ischaemia during exercise, especially in the subendocardium. ${ }^{18}$ Coronary microvascular flow impairment is a potent long-term predictor of adverse LV remodelling and systolic dysfunction, which may exacerbate energy deficiency. ${ }^{19}$ To test the HCM-induced "stunning" hypothesis, exacerbation of energy deficiency during exercise should be formally demonstrated in HCM patients. Moreover, a comparison should be made between the cellular consequences of exercise-induced energy deficiency and ischaemic stunning. If these two conditions prove mechanistically similar, HCM animals may prove particularly tractable models in which to study the complexities of stunning ${ }^{14}$ and the sizeable stunning literature may be reciprocally informative regarding the pathogenesis of $\mathrm{HCM}^{15}$ exercise in HCM may induce contractile dysfunction, which is associated ${ }^{13}$ with
Repetitive episodes of fatigue-inducing long term ventricular dysfunction and may portend ominous outcomes. There is, however, little evidence at this stage that symptom-limited echocardiographic or radionuclide exercise testing is warranted in identifying high-risk patients as a genetic surrogate or for clinical prognostication purposes. Nevertheless, these studies provide a good rationale to investigate the role of exercise-induced exacerbation of energy deficiency and stunning in HCM. They also provide another rationale to warn $\mathrm{HCM}$ patients to be wary of extreme recreational or professional exercise.

Competing interests: None declared.

Heart 2008;94:1251-1253.

doi:10.1136/hrt.2007.123166

\section{REFERENCES}

1. Ashrafian H, Watkins $\mathrm{H}$. Reviews of translational medicine and genomics in cardiovascular disease: new disease taxonomy and therapeutic implications cardiomyopathies: therapeutics based on molecular phenotype. J Am Coll Cardiol 2007;49:1251-64.

2. Maron BJ. Sudden death in young athletes. N Engl J Med 2003;349:1064-75

3. Nagueh SF, Mahmarian JJ. Noninvasive cardiac imaging in patients with hypertrophic cardiomyopathy. J Am Coll Cardiol 2006;48:2410-22.

4. Frenneaux MP. Assessing the risk of sudden cardiac death in a patient with hypertrophic cardiomyopathy. Heart 2004;90:570-5.

5. Maron BJ, Thompson PD, Ackerman MJ, et al. Recommendations and considerations related to preparticipation screening for cardiovascular abnormalities in competitive athletes: 2007 update: a scientific statement from the American Heart Association Council on Nutrition, Physical Activity, and Metabolism: endorsed by the American College of Cardiology Foundation. Circulation 2007; 115:1643-455.

6. Sakata K, Ino H, Fujino N, et al. Exercise-induced systolic dysfunction in patients with non-obstructive hypertrophic cardiomyopathy and mutations in the cardiac troponin genes. Heart 2008;94:1282-7.

7. Ciampi 0, Betocchi S, Lombardi R, et al. Hemodynamic determinants of exercise-induced abnormal blood pressure response in hypertrophic cardiomyopathy. J Am Coll Cardiol 2002;40:278-84.

8. Campbell R, Manyari DE, McKenna WJ, et al. What is the mechanism of abnormal blood pressure response on exercise in hypertrophic cardiomyopathy? J Am Coll Cardiol 2003;41:2102.

9. Frenneaux MP, Counihan PJ, Caforio AL, et al. Abnormal blood pressure response during exercise in hypertrophic cardiomyopathy. Circulation 1990;82:1995-2002

10. Counihan PJ, Frenneaux MP, Webb DJ, et al. Abnormal vascular responses to supine exercise in hypertrophic cardiomyopathy. Circulation 1991;84:686-96.

11. Ciampi 0, Betocchi S, Violante A, et al. Hemodynamic effects of isometric exercise in hypertrophic cardiomyopathy: Comparison with normal subjects. J Nucl Cardiol 2003;10:154-60.

12. Okeie K, Shimizu M, Yoshio H, et al. Left ventricular systolic dysfunction during exercise and dobutamine stress in patients with hypertrophic cardiomyopathy. J Am Coll Cardiol 2000;36:856-63.

13. Pelliccia F, Cianfrocca C, Pristipino C, et al. Cumulative exercise-induced left ventricular systolic and diastolic dysfunction in hypertrophic cardiomyopathy. Int J Cardiol 2007;122:76-8.

14. Bolli R. Basic and clinical aspects of myocardial stunning. Prog Cardiovasc Dis 1998;40:477-516. 
15. Kloner RA, Jennings RB. Consequences of brief ischemia: stunning, preconditioning, and their clinical implications: part 1. Circulation 2001;104:2981-9.

16. Javadpour MM, Tardiff JC, Pinz I, et al. Decreased energetics in murine hearts bearing the R920 mutation in cardiac troponin T. J Clin Invest 2003:112:768-75
17. Frey N, Brixius K, Schwinger RH, et al. Alterations of tension-dependent ATP utilization in a transgenic rat model of hypertrophic cardiomyopathy. J Biol Chem 2006:281:29575-82.

18. Petersen SE, Jerosch-Herold M, Hudsmith LE, et al. Evidence for microvascular dysfunction in hypertrophic cardiomyopathy: new insights from multiparametric magnetic resonance imaging. Circulation

2007:115:2418-25.

19. Olivotto I, Cecchi F, Gistri R, et al. Relevance of coronary microvascular flow impairment to long-term remodeling and systolic dysfunction in hypertrophic cardiomyopathy. J Am Coll Cardiol 2006;47:1043-8.

\section{Images in cardiology}

\section{Apical hypertrophic cardiomyopathy and left ventricular non-compaction: two faces of the same disease}

A 15-year-old male came to our clinic because his father had died suddenly with a previous diagnosis of apical hypertrophic cardiomyopathy at age 38. His father's records said he had congestive heart failure, a maximal left ventricular wall thickness of $28 \mathrm{~mm}$, biventricular systolic dysfunction and left atrial hypertrophy on echocardiography. A female cousin of the father had had a heart transplant because of restrictive hypertrophic cardiomyopathy with severe heart failure (the explanted heart showed hypertrophy, myocyte disarray and fibrosis). At 18 years of age, our patient was asymptomatic on normal physical examination. The ECG demonstrated sinus rhythm with biventricular hypertrophy. The echocardiogram showed a normal wall thickness in the basal and mid-left ventricular segments and prominent trabeculations in the apical posterior, inferior and lateral walls. The diagnosis of left ventricular non-compaction was confirmed on cardiac magnetic

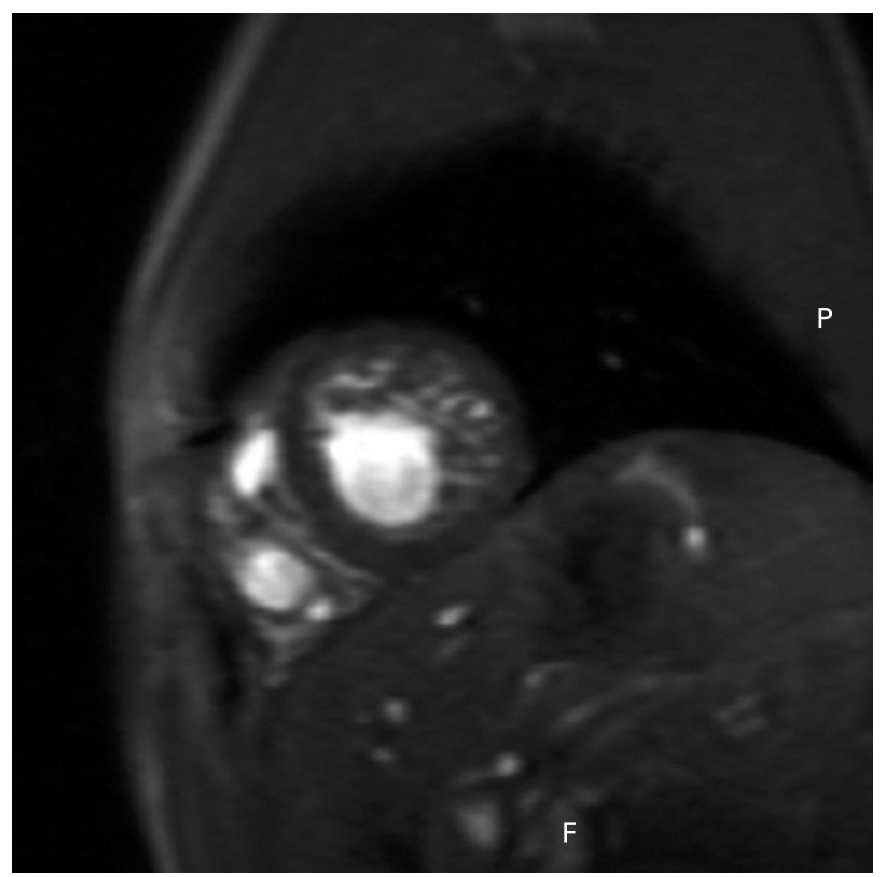

Panel A

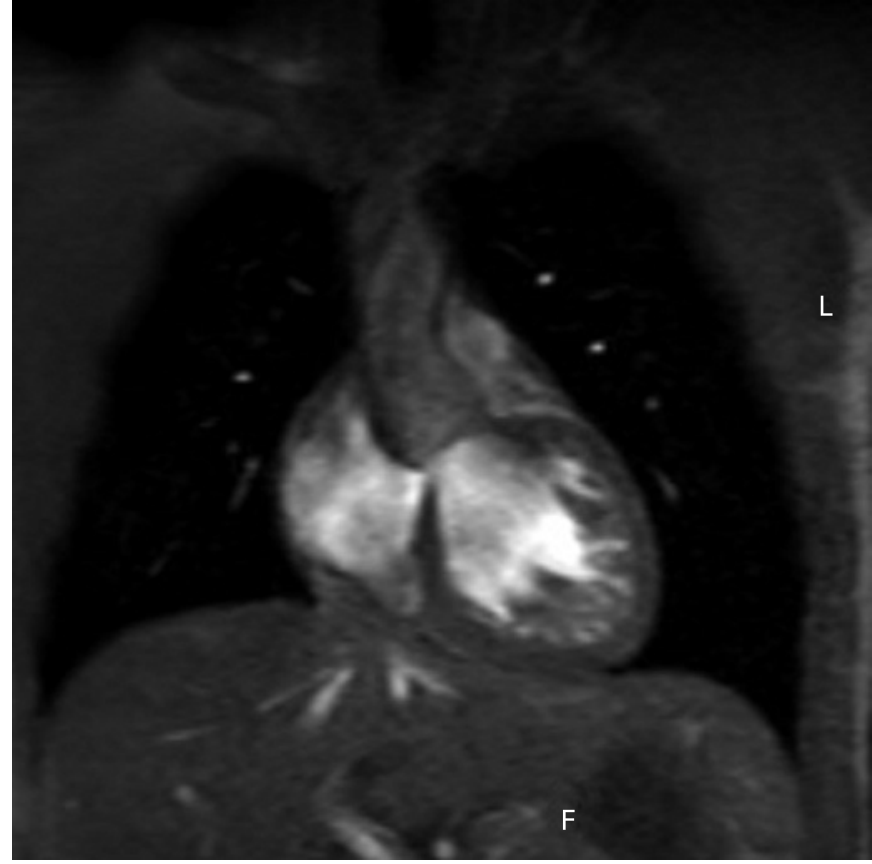

Panel B

resonance imaging (panels $\mathrm{A}$ and $\mathrm{B}$ ). Holter monitoring was normal. Exercise echocardiography demonstrated an increase in ejection fraction (from $60 \%$ to $68 \%$ ) and a rise in systolic blood pressure (from $120 \mathrm{~mm} \mathrm{Hg}$ to $170 \mathrm{~mm} \mathrm{Hg}$ ). Genetic testing demonstrated a heterozygous mutation from guanine to adenine at nucleotide 2263 of the ACTC gene that causes a change from glutamic acid to lysine at the amino acid 101 of the protein $(\mathrm{E} 101 \mathrm{~K})$. An association of this mutation with noncompaction and septal defects has been subsequently confirmed in six families from Galicia, Spain, and two from SwitzerlandGermany. These and other cases demonstrate the association between mutations in cardiac sarcomeric protein genes and left ventricular non-compaction.

\section{Monserrat, R Barriales-Villa, M Hermida-Prieto}

Correspondence to: Dr Lorenzo Monserrat, Instituto de Investigación Biomédica de A Coruña (INIBIC), CHU Juan Canalejo, As Xubias 84, A Coruña 15006, Spain; Lorenzo_monserrat@canalejo.org

Competing interests: $L M$ and MH-P have shares in Health In Code SL. LM is employed by Health In Code SL. MH has received research funding from Health In Code SL.

Heart 2008;94:1253. doi:10.1136/hrt.2008.154047 